\title{
DESIGN AND SIMULATION OF ADVANCED BUFFERED AMPLIFIER FOR LOW POWER BIOMEDICAL APPLICATIONS
}

\author{
Sarah Khwaja \\ Bachelor of Engineering (Gold Medalist), Jamia Millia Islamia, India
}

\begin{abstract}
In this paper, Advanced Buffered Amplifier is designed using Cascode Operational transconductance amplifier at $45 \mathrm{~nm}$ technology using $0.9 \mathrm{~V}$. The proposed structure uses carbon nanotube field effect transistors. It is observed that the proposed Buffered Amplifier has Bandwidth of $467 \mathrm{GHz}$, Power Dissipation of 0.35 $u W$, Output Impedance of $1.6 \mathrm{~K} \Omega$, Input Impedance of $6.5 \mathrm{~K} \Omega$, Phase Margin of 187 degree. Further, in the proposed structure, Bandwidth is $29.72 \%$ higher, Power Dissipation is $42.85 \%$ lower, Output Impedance is $11.11 \%$ lower, Input Impedance is $8.3 \%$ higher as compared with its CMOS counterpart. The proposed advanced buffered amplifier is electronically tunable. It can be used for Low Power Biomedical Applications due to low power, low voltage and low amplitude of signal.
\end{abstract}

Keywords: CMOS, CNTFET, COTA, Power consumption, Buffered Amplifier

Cite this Article: Sarah Khwaja, Design and Simulation of Advanced Buffered Amplifier for Low Power Biomedical Applications, International Journal of Advanced Research in Engineering and Technology (IJARET), 11 (1), 2020, pp 23-29. $\mathrm{http} / /$ iaeme.com/Home/issue/IJARET?Volume=11\&Issue $=1$

\section{INTRODUCTION}

Cascode Operational transconductance amplifier (COTA) is a new class of O.T.A. It has improved gain (due to Band output resistance) and bandwidth due to reduced Miller capacitance [1-2]. In order to ensure further improvement in Cascode COTA performance, Carbon Nanotube Field Effect Transistors (CNTFETs) based Circuits that promise to deliver much better performance than existing CMOS based Cascode Operational transconductance amplifiers is proposed [2-4]. CNTFET technology can easily club with the bulk CMOS technology on a single chip and utilizes the same infrastructure at $45 \mathrm{~nm}$ [5-7].

\section{DESIGN OF ADVANCED BUFFERED AMPLIFIER}

A buffer amplifier (sometimes simply called a buffer) is one that provides electrical impedance transformation from one circuit to another, with the aim of preventing the signal source from being affected by the load. 

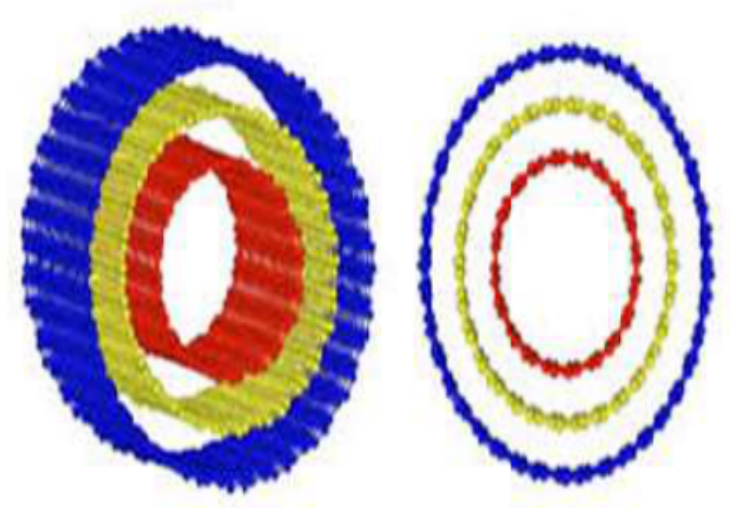

Figure 1.The off-axis and top views of MWCNTs with three walls

\section{PROPOSED COTA BASED ADVANCED BUFFERED AMPLIFIER}

The Cascode Operational Transconductance Amplifier circuit is used to design Buffered amplifier. The proposed Buffered amplifier is simulated using $0.9 \mathrm{~V}$ at $45 \mathrm{~nm}$. In the Cascode Operational transconductance amplifier based Advanced Buffered Amplifier, there is no need of negative feedback and feedback resistor. Further the proposed Amplifier consumes very low power and simple circuitry. Furthermore, the proposed advanced buffered amplifier is electronically tunable [8-12].

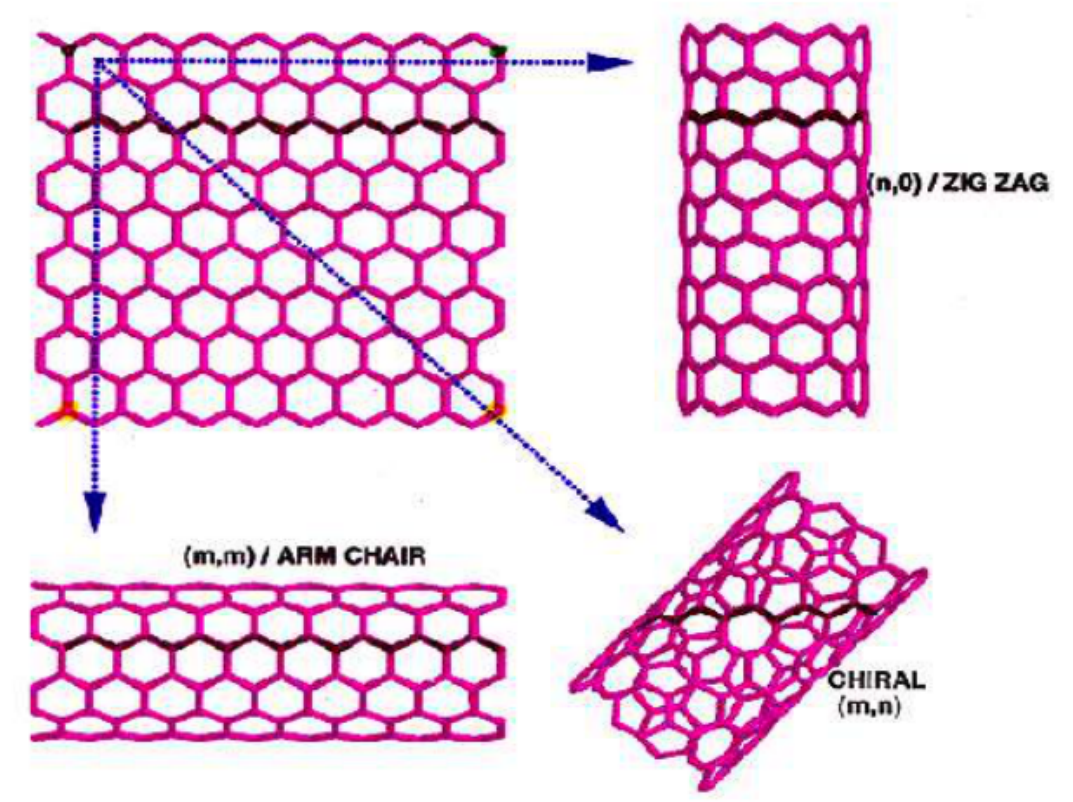

Figure 2. Different configurations of Single wall CNT.

The carbon nanotubes exist in two forms: (i) Single wall carbon nanotube (SWCNT) and (ii) multiwall carbon nanotube as shown in Figure 1. SWCNT are actually tubes of graphite that are normally capped at the ends. They can be visualized as a layer of graphite rolled into a seamless cylinder [8-9]. Their diameter is around $1 \mathrm{~nm}$ and length a few microns. They are superior to MWCNT, however, are costlier. MWCNT appear like a coaxial assembly of SWCNTs, like a coaxial cable. They diameter of MWCNT ranges from 5-50 nm and the inter layer spacing is $3.4 \AA$. They are easy to produce in large quantity. However, the structure is 
complex and the structural imperfections may diminish their unique properties [10]. The wrapping of graphite sheets in SWCNT can be represented by a pair of indices $(n, m)$, called as the chirality vector or roll-up vector, as shown in Figure 1. There are three types of SWCNTs based on the chiral vector and chiral angle $(\theta)$.

Figure 3. shows the schematics of one of the important application of CNT, that is, carbon nanotube field effect transistor (CNTFET). In a CNTFET, the channel is made up of parallel combination of SWCNTs. The important advantages of CNTFET include 1D ballistic transport of charge carriers, high mobility, large drive current and very low power consumption [11]. Nanotube diameter and chiral angles are given as [12-22]

$$
\begin{gathered}
d_{t}=\frac{\sqrt{3 a_{c-c} \sqrt{m^{2}+m n+n^{2}}}}{\pi} \\
\theta=\tan ^{-1} \frac{\sqrt{3 n}}{2 m+n}
\end{gathered}
$$
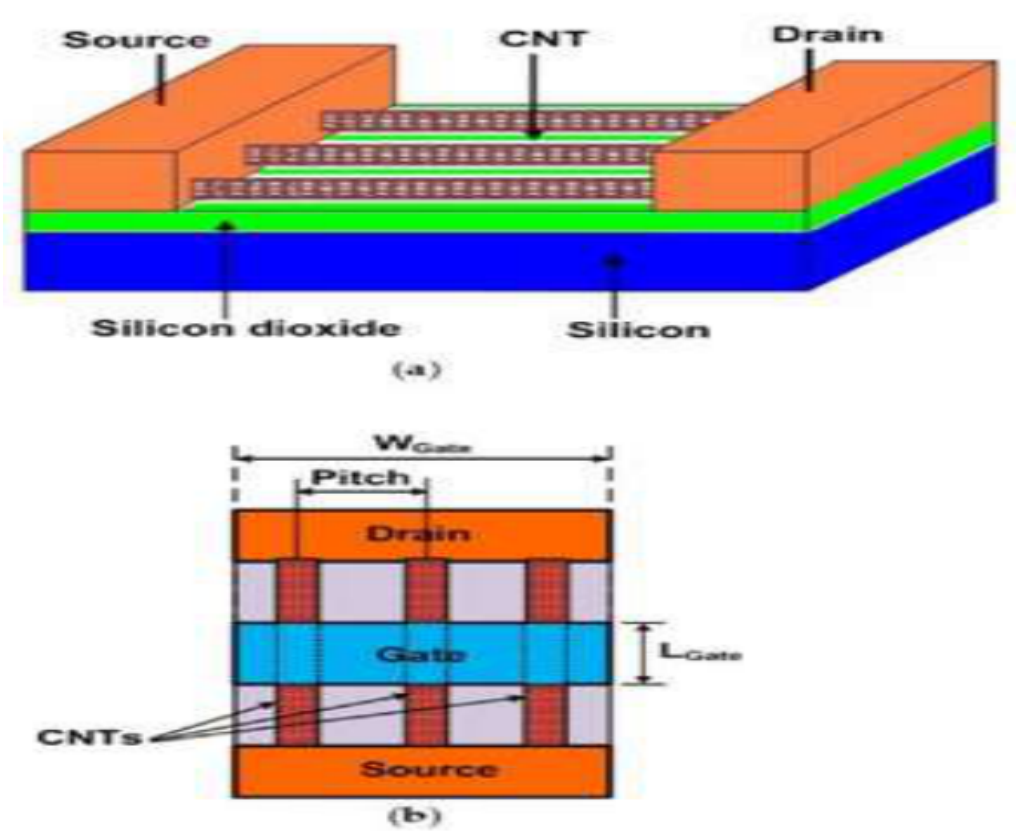

Figure 3. Carbon nanotube field effect transistor (CNTFET) (a) Schematic; (b) Top-View

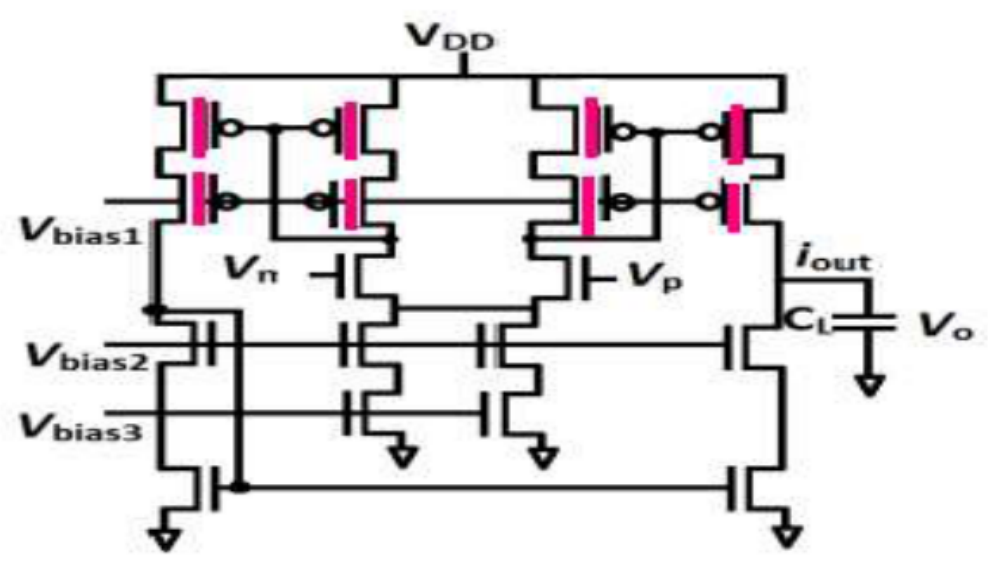

Figure 4. Cascode Operational Transconductance Amplifier [9] 


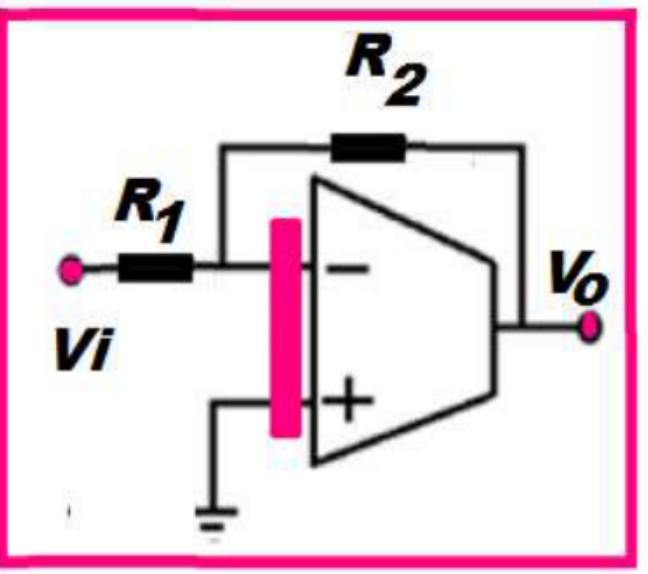

Figure 5. Advanced Buffered amplifier

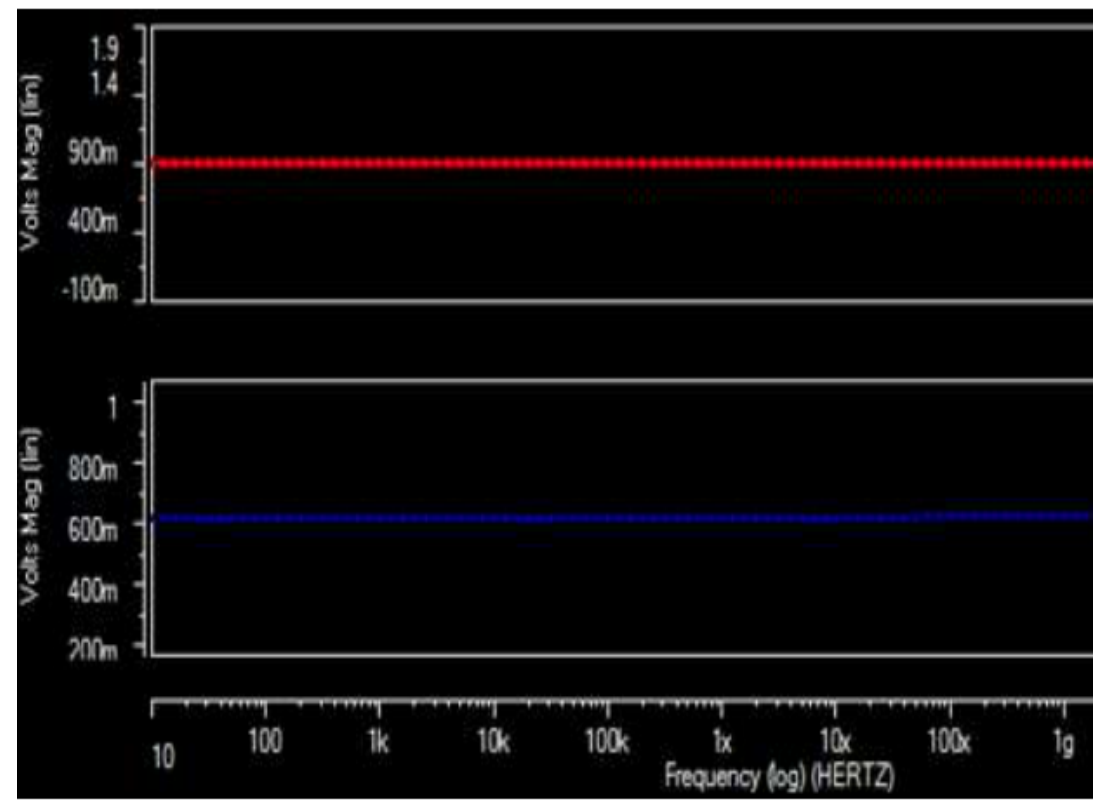

Figure 6. Frequency response of Proposed Buffered Amplifier

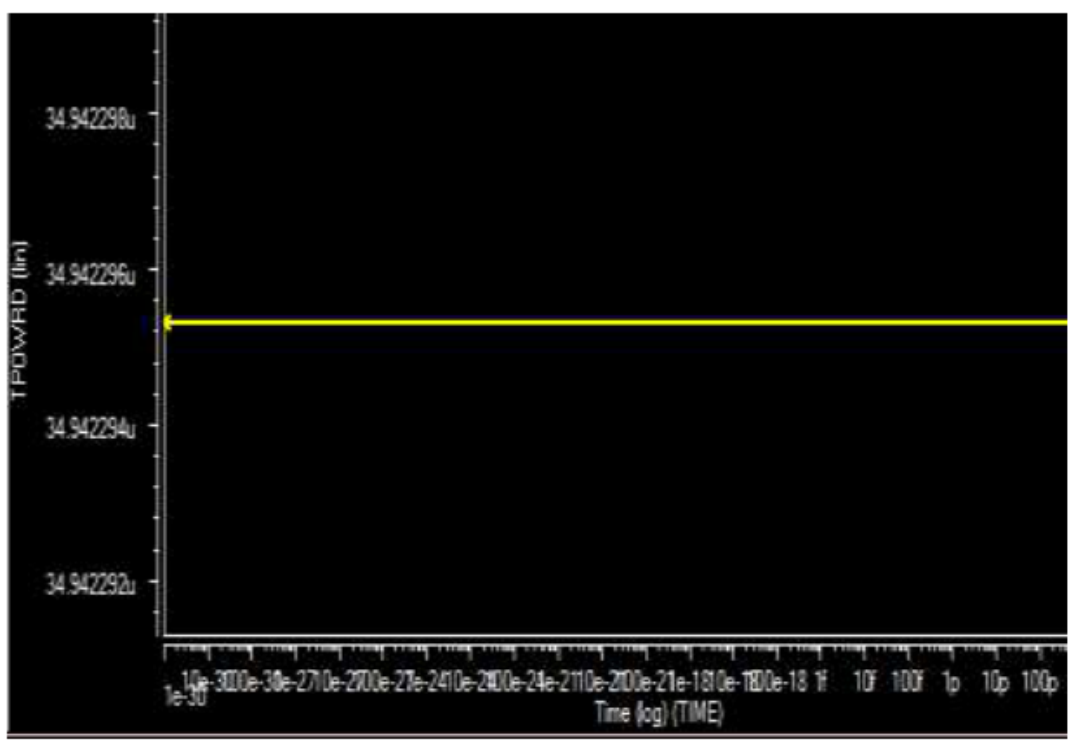

Figure 7. Average Power of Proposed Buffered amplifier 
Design and Simulation of Advanced Buffered Amplifier for Low Power Biomedical Applications

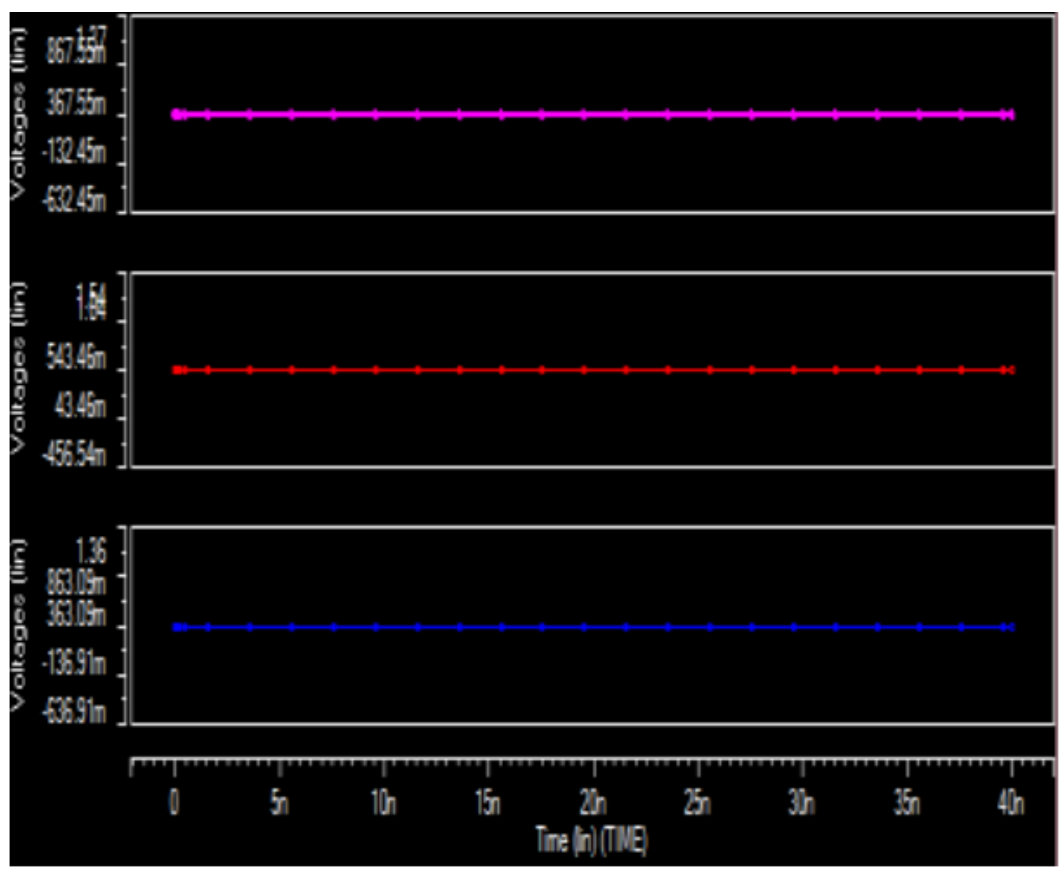

Figure 8. Biasing Voltages used in Proposed Buffered amplifier

Table 1 Performance Measuring Parameters at $0.9 \mathrm{~V}$ using $45 \mathrm{~nm}$ technology node

\begin{tabular}{|c|l|c|c|}
\hline S. No. & \multicolumn{1}{|c|}{ Parameter } & $\begin{array}{c}\text { Proposed } \\
\text { Buffered } \\
\text { Amplifier }\end{array}$ & $\begin{array}{c}\text { Conventional } \\
\text { Buffered Amplifier }\end{array}$ \\
\hline 1 & Bandwidth & $467 \mathrm{GHz}$ & $360 \mathrm{GHz}$ \\
\hline 2 & Power Dissipation & $0.35 \mathrm{uW}$ & $0.63 \mathrm{uW}$ \\
\hline 3 & Output Impedance & $1.6 \mathrm{~K}$ & $1.8 \mathrm{~K}$ \\
\hline 4 & Input Impedance & $6.5 \mathrm{~K}$ & $6 \mathrm{~K}$ \\
\hline 5 & Phase Margin & 1870 & 1860 \\
\hline
\end{tabular}

\section{CONCLUSION}

In this work, Simulation study of Advanced Buffered Amplifier for Low Power Biomedical Applications is proposed. The proposed structure uses carbon nanotube field effect transistor. In the proposed structure, Bandwidth is $29.72 \%$ higher, Power Dissipation is $42.85 \%$ lower, Output Impedance is $11.11 \%$ lower, Input Impedance is $8.3 \%$ higher as compared with its CMOS counterpart. Furthermore, the proposed advanced buffered amplifier is electronically tunable. It can be used for Low Power Biomedical Applications.

\section{REFERENCES}

[1] Toumazou, F. D. Lidgey, and D. G. Haigh, "Analog IC Design: The Current Mode Approach" London, U.K.: Peregrinus, 1990.

[2] RCA Solid-state Division, Data Book, "Linear Integrated Circuits", File No.475, Mar.1975.

[3] Åkerberg, Dag, and Kare Mossberg, "A Versatile Active RC Building Block with Inherent Compensation for the Finite Bandwidth of the Amplifier," IEEE Transactions on Circuits and Systems, vol. 21, no. 1 pp. 75-78, 1974. 
[4] Xuguang Zhang and Ezz I. El-Masry,"A Novel CMOS COTA Based on Body-Driven MOSFETs and its Applications in COTA-C Filters", IEEE Transactions on Circuits and Systems-I: Regular Papers, vol. 54, no. 6, pp 1204-1212, June 2007.

[5] Sarah Khwaja, "Simulation study of Advanced Inverting Amplifier for Low Power Biomedical Applications", International Journal of Multidisciplinary Educational Research, Vol 8, Issue 11(4), Nov. 2019.

[6] S. A. Loan, M. Nizamuddin et.al., "Band Performance Carbon Nanotube Based Cascode Operational Transconductance Amplifiers", World Congress on Engineering, Vol 1, July $2-4,2014$.

[7] M. Nizamuddin, S. A. Loan, et.al., "Design, Simulation and The Comparative Analysis of CNTFET Based Multistage Operational Amplifiers", Journal of Nanoelectronics and Optoelectronics, Vol-12, 2017.

[8] S. A. Loan, M. Nizamuddin et.al., "Design and Comparative Analysis of Band Performance Carbon Nanotube-Based Cascode Operational transconductance amplifiers", NANO: World Scientific Publisher, Vol. 10, No. 3, 2015.

[9] M. Nizamuddin, S. A. Loan, et.al., "Design, Simulation and Comparative Analysis of CNT based Cascode Cascode Operational Transconductance Amplifiers", Volume 26, Number 39, 02 , Nanotechnology, IOP Publishing Ltd ,October 2015.

[10] S. A. Loan et al., Design of a novel Band Gain Carbon Nanotube Based Cascode Operational Transconductance Amplifier, Proc. IMECS PP. 797-800, 2014.

[11] M. Nizamuddin, et.al., "Carbon Nanotube Based Three Stage Operational Amplifiers, A Simulation Study", Journal of Materials Today Proceedings, Volume 3, Issue 2, Pages 449-453, 2015.

[12] Fahad Ali Usmani, et.al., "Carbon Nanotube Field Effect Transistors for High Performance Analog Applications: An Optimum Design Approach", Microelectronics Journal, 41, 2010.

[13] J.Deng, H.S.P.Wong, "A Compact SPICE Model for Carbon Nanotube Field Effect Transistors Including Non-Idealities and its Application Part II:" IEEE Transactions on Electron Devices 54, 2007.

[14] Sarah khwaja, "Design and Simulation of High Performance Low Power Analog Filters for Biomedical Applications at $90 \mathrm{~nm}$ Technology Node", International Journal of Multidisciplinary Educational Research, Volume 8, Issue 12(2), December, 2019.

[15] J.Appenzeller, "Carbon Nanotubes for High Performance Electronics", Proceedings of the IEEE 96(2), 2008.

[16] Sarah Khwaja, "Design and Simulation of High Performance High Pass Filter at $45 \mathrm{~nm}$ Technology Node", International Journal of Innovative Research in Electrical, Electronics, Instrumentation and Control Engineering Vol. 7, Issue 7, July 2019.

[17] Sarah Khwaja, "Simulation Study of High Performance Low Pass filter at Nanoscale", International Journal of Advanced Research in Computer and Communication Engineering Vol. 8, Issue 8, Aug 2019.

[18] Sarah Khwaja, "Design and Performance Analysis of Low Power Band Pass Filter at 32 $\mathrm{nm}$ Technology", International Journal of Innovative Research in Electrical, Electronics, Instrumentation and Control Engineering Vol. 7, Issue 9, September 2019.

[19] Faisal Bashir, Sajad A Loan, M Nizamuddin, et.al., " A Novel High Performance Nano Scaled Dopingless Lateral PNP Transistor on Silicon on Insulator", Proceedings of the International Multi Conference of Engineers and Computer Scientists, 2014. 
Design and Simulation of Advanced Buffered Amplifier for Low Power Biomedical Applications

[20] Sarah Khwaja, "Design and Performance Analysis of Low Power Band Stop Filter, International Journal of Advance Research in Engineering and Technology, 10 (6), 2019, pp 16-21.

[21] Sajad A Loan, Faisal Bashir, M Rafat, M Nizamuddin, Asim M Murshid, Abdul Rahman M Alamoud, Shuja A Abbasi, "A Novel High Performance Nanoscaled Dual Oxide Doping Less Tunnel Field Effect Transistor", Proceedings of the World Congress on Engineering, 2014.

[22] M Nizamuddin, "Design \& Performance Analysis of Instrumentation Amplifier at Nanoscale”, Int J Adv Res Ideas Innov Technol, Volume 3, Issue 1, Pages 793-798, 2017.

[23] Muhammad Masud, et.al., "Design of Voltage Mode Electronically Tunable First Order All Pass Filter in 0.7 V 16 nm CNFET Technology", Electronics MDPI, Open Access Journal, 2019 